\title{
A comparison of cellulose acetate immunofixation with polyacrylamide gel electrophoresis for the detection of oligoclonal bands in unconcentrated cerebrospinal fluid
}

\author{
FC GOODLAND, EJ THOMPSON
}

From the Department of Chemical Pathology (NHS), General Hospital Southampton S09 4XY, and Clinical Neurochemistry, Institute of Neurology, Queen Square, London WC1N $3 B G$

SUMMARY Two methods of electrophoresis for the detection of oligoclonal bands in unconcentrated CSF were compared. A sample of 98 routine CSFs yielded 18 positives by polyacrylamide gel electrophoresis (PAGE) while cellulose acetate electrophoresis with immunofixation (CAIF) gave 13 positives (72\% of the PAGE findings). Despite the loss of sensitivity the cellulose acetate electrophoresis was easier to interpret and more suited to a routine hospital laboratory.

Electrophoresis of cerebrospinal fluid (CSF) to detect oligoclonal immunoglobulins is a useful test in the investigation of neurological disease. Polyacrylamide, ${ }^{1}$ agar $^{23}$ and agarose ${ }^{3-6}$ gels and cellulose acetate $^{78}$ have all been used as support media with varying success. Cellulose acetate is reported to have poorer resolution than the gels. ${ }^{8}$ Many investigators have concentrated their CSF samples before electrophoresis although it has been shown this may alter the electrophoretic pattern. ${ }^{7}$ Thompson ${ }^{1}$ therefore applied relatively large volumes of CSF directly to PAG disc electrophoresis. This technique gives good results in experienced hands but those unfamiliar with PAGE may have difficulty with the interpretation.

Electrophoresis of unconcentrated CSF on cellulose acetate is feasible when Nigrosine stain is used. ${ }^{79}$ However $\gamma$ globulins stain weakly and $\beta$ and $\gamma$ trace globulins tend to confuse the picture when looking for oligoclonal bands. ${ }^{368}$ Immunofixation with antihuman IgG antisera further intensifies the $\gamma$ globulin staining and removes interference from other proteins. ${ }^{610}$

The detection of oligoclonal bands in unconcentrated CSF on cellulose ace tate with immunofixation was compared with PAGE.

\section{Material and methods}

SAMPLES

Ninety-eight unselected routine CSF samples on Accepted for publication 23 May 1983 which PAGE had been performed at the Institute of Neurology were sent to Southampton for electrophoresis on cellulose acetate. A further selected group of 13 positive and two negative CSF samples was later sent. All samples were analysed and evaluated blind.

POLYACRYLAMIDE GEL ELECTROPHORESIS

PAGE was performed by the method of Davis. ${ }^{11}$ Using $7 \%$ polyacrylamide but without a stacking gel, Thompson, ${ }^{1}$ two gels were run for each CSF. One was loaded with $100 \mu \mathrm{g}$ total protein and the other with $200 \mu \mathrm{g}$. The first gel was stained with Coomassie blue and the second with naphthalene black. Immunoglobulin bands were identified by their greater relative affinity for Coomassie blue than naphthalene black.

\section{CELLULOSE ACETATE ELECTROPHORESIS}

A method for detecting Bence Jones protein in unconcentrated urine ${ }^{10}$ was modified by using anti $\gamma$ chain antiserum in place of anti-light chain antiserum.

Cellulose acetate electrophoresis was performed on Titan III mylar backed cellulose acetate plates with a Tris-barbital buffer $\mathrm{i}=0.05, \mathrm{pH}=8.6$ using standard applicators and other electrophoresis equipment all from Helena, Beaumont Texas, USA.

Prior to soaking in electrophoresis buffer, a line is drawn on the mylar backing of immunofixation plates in waterproof ink $32 \mathrm{~mm}$ from the cathodic end. This can be seen through wet cellulose acetate 


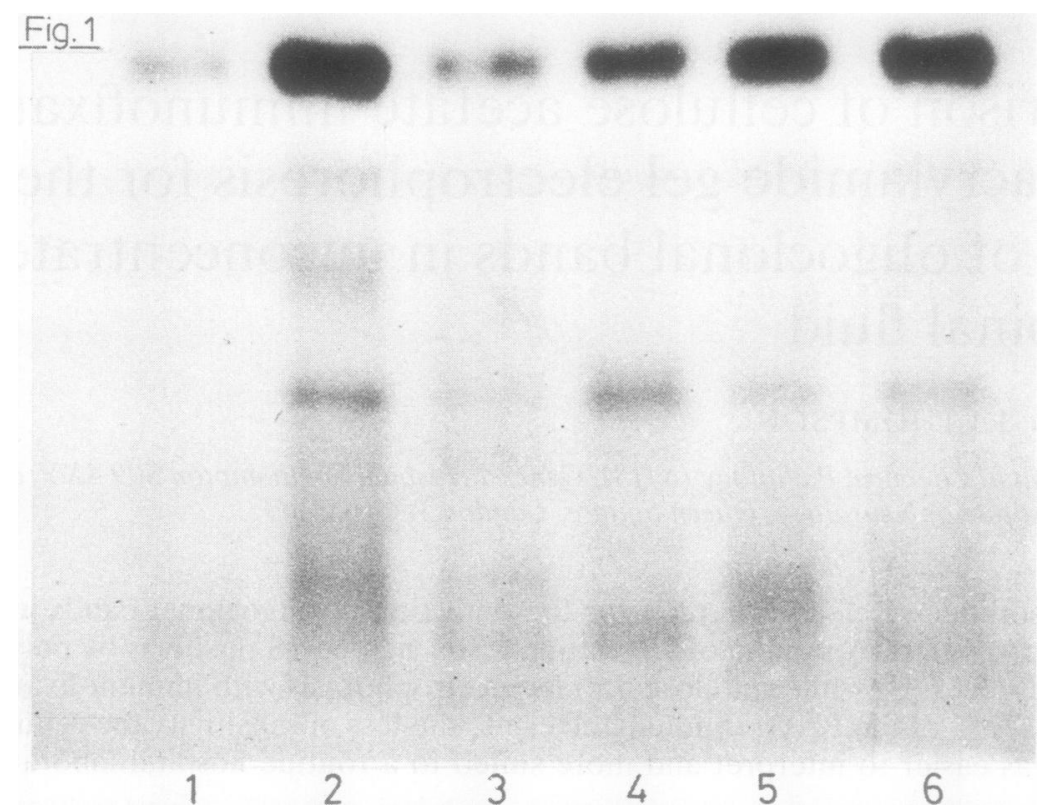

Fig. 1 Cellulose acetate electrophoresis stained for total protein. Samples from left to right 1, 3-5 positive, 2, 6 negative.

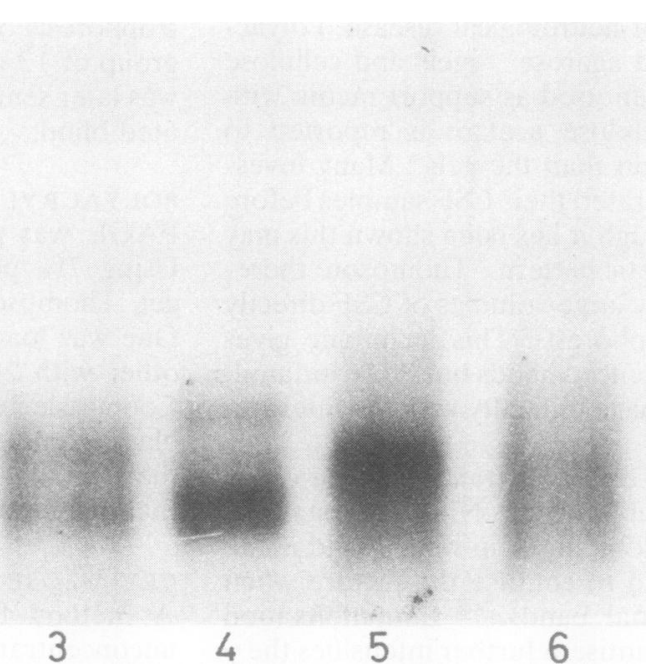

Fig. 2 Cellulose acetate electrophoresis after immunofxation. Samples from left to right 1, 3-5 positive, 2, 6 negative.

and guides the positioning of the antiserum strip. CSF $(5 \mu \mathrm{l})$ is placed in each sample applicator well, but by a double application with the Super $Z$ applicator only $0.3 \mu \mathrm{l}$ of CSF is loaded onto the plate. Duplicate sets of plates were run. After electrophoresis at $180 \mathrm{~V}$ for $15 \mathrm{~min}$ the plates to be stained for total protein were fixed in TCA $100 \mathrm{~g} / \mathrm{l}$ for $30 \mathrm{~min}$ before staining in Nigrosine $50 \mathrm{mg} / \mathrm{l}$ in acetic acid $50 \mathrm{ml} / 1$ for $30 \mathrm{~min}$. Following a $30 \mathrm{~min}$ N wash in dilute acetic acid $50 \mathrm{ml} / \mathrm{l}$, the plates were $\mathrm{W}$ blotted and air dried.

The plates for immunofixation were placed in humidified chamber after electrophoresis. Antihuman $\gamma$ chain antiserum Dako (Mercia-Brocades Ltd, $\stackrel{\oplus}{\rightarrow}$ West Byfleet, Surrey) was diluted $1 / 3$ in physiologi- $\square$ cal saline. Dilute antiserum $(250 \mu \mathrm{l})$ completely 
moistens a $25 \times 55 \mathrm{~mm}$ strip of cellulose acetate (Cellogram) which was then placed over the $\gamma$ region of the electrophoresis plates-that is, anodically of the line previously drawn on the back. After 15 min the antiserum strips were discarded and the electrophoresis plates washed for one hour in detergent buffer, $\mathrm{NaCl} 9.0 \mathrm{~g} / \mathrm{l}, \mathrm{Na}$ barbitone $5.0 \mathrm{~g} / \mathrm{l}$, Triton X $1001.0 \mathrm{~g} / \mathrm{l}$, to remove unfixed proteins. The plates were then stained with Nigrosine as described for the total protein plates. The cellulose ace tate plates were viewed dry, uncleared by transillumination.

\section{Results}

Unconcentrated CSF when stained for total protein after electrophoresis gives a clearly discernible pattern from the albumin to $\beta$ globulin region but $\gamma$ region is diffuse and relatively poorly stained. Only the more intense oligoclonal bands can be unequivocally identified. (Fig 1) Immunofixation intensifies and clarifies staining in the globulin region (Fig 2) so that a single band can be taken as evidence of oligoclonal antibodies. Of 98 unselected routine CSF samples investigated 18 had oligoclonal bands by PAGE while only 13 (72\% of PAGE findings) showed banding on CAIF. Of an additional selected group of 13 positive and two negative CSFs by PAGE, nine ( $69 \%$ of PAGE findings) positives were obtained by CAIF. At no time did CAIF give a positive result with a sample that was negative by PAGE.

\section{Discussion}

These results are consistent with the greater resolving power of PAGE over cellulose acetate in the detection of oligoclonal bands in CSF. However many laboratories do not have the time, expertise or equipment to run PAGE, while most laboratories already run some cellulose acetate electrophoresis and hence have the potential to run CAIF. Another alternative would be agarose gel electrophoresis followed by immunofixation which takes slightly longer and is slightly more involved than CAIF. ${ }^{4}$ There is moreover a considerable saving of time with CAIF, the whole procedure taking only $3 \frac{1}{2} \mathrm{~h}$. There is no need to concentrate samples and as little as $5 \mu \mathrm{l} \mathrm{CSF}$ may be used. The interpretation of CAIF is easier than with PAGE. Although the detection rate for CAIF was lower than for PAGE there were no false positives.

The CSF: serum IgG: albumin ratio has also been used for detection of MS. We cannot comment on its effectiveness relative to CAIF as no comparison has been made in this series. It has been shown that the
CSF: serum IgG: albumin ratio is inferior to PAGE in the detection of MS producing both false positives and negatives. ${ }^{12}$ The ratio requires four immunological assays on each patient involving considerable analytical time, reagent costs and/or capital expenditure. Moreover the experience of the Institute of Neurology is that despite every effort only $20 \%$ of CSF samples arrive accompanied by a blood sample, thus only the CSF IgG: albumin or CSF IgG: total protein can be measured.

We therefore consider that CAIF would be a simple and useful additional technique for busy routine and smaller laboratories.

We thank Dr JE Middleton, Consultant Chemical Pathologist, Southampton for his advice and encouragement.

\section{References}

' Thompson EJ, Kaufman P, Shortman RC, Rudge P, McDonald WI. Oligoclonal immunoglobulins and plasma cells in spinal fluid of patients with multiple sclerosis. Br Med J 1979;i:16-7.

${ }^{2}$ Laterre EC, Heremaus JE. Electrophoretic morphology of gamma globulins in cerebrospinal fluid of multiple sclerosis and other diseases of the nervous system. Neurology 1970;20:982-90.

${ }^{3}$ Link $\mathrm{H}$. Comparison of electrophoreis on agar gel and agarose gel in the evaluation of gamma globulin abnormalities in cerebrospinal fluid and serum in multiple sclerosis. Clin Chim Acta 1976;46:383-9.

4 Keir G, Walker RW, Johnson MH, Thompson EJ. Nitrocellulose immunofixation following agarose electrophoresis in the study of immunoglobulin $\mathrm{G}$ subgroups in unconcentrated cerebrospinal fluid. Clin Chim Acta 1982;121:231-6.

${ }^{5}$ Gerson B, Krolikowski FJ, Gerson IM. Two agarose electrophoretic systems for demonstration of oligoclonal bands in cerebrospinal fluid compared. Clin Chim Acta 1980;26:343-5.

- Cawley LP, Minard BJ, Tourtellotte WW, Ma BI, Chelle C. Immunofixation electrophoretic techniques applied to identification of proteins in serum and cerebrospinal fluid. Chin Chim Acta 1976;22:1262-8.

' Glasner H. Microzone electrophoresis of unconcentrated and concentrated CSF. J Neurol 1978;218:73.

${ }^{8}$ Vandvik B and Skrede S. Electrophoretic examination of cerebrospinal fluid protein in multiple sclerosis and other neurological diseases. Eur Neurol 1973;9:224-41.

' Sherwin MF, Moore GH. Microzone electrophoresis of unconcentrated cerebrospinal fluid using cellulose acetate strips and nigrosine dye. Am J Clin Pathol 1971;55:705-12.

${ }^{10}$ Goodland FC. Detection limits for protein after electrophoresis on cellulose acetate. Ann Clin Biochem 1982;19:117-9.

" Davis BJ. Disc electrophoresis-II Method and application to human serum proteins. Ann NY Pract Sci 1964;121:404-27.

12 Thompson EJ, Riches PG, Kohn J. Antibody synthesis within the central nervous system: comparisons of CSF IgG indices and electrophoresis. J Clin Pathol 1983;36:312-5.

Requests for reprints to: Mr FC Goodland, Department of Chemical Pathology (NHS), South Laboratory Block, General Hosptial, Southampton S09 4XY, England. 